IHES $/ \mathrm{P} / 93 / 48$

CPT-93/P.2941

\title{
Testing for Preferred-Frame Effects in Gravity with Artificial Earth Satellites
}

\author{
Thibault Damour \\ Institut des Hautes Etudes Scientifiques, 91440 Bures sur Yvette, France \\ and Département d'Astrophysique Relativiste et de Cosmologie, Observatoire de Paris, \\ Centre National de la Recherche Scientifique, 92195 Meudon, France \\ Gilles Esposito-Farèse \\ Centre de Physique Théorique, Centre National de la Recherche Scientifique, \\ Luminy, Case 907, 13288 Marseille Cedex 9, France
}

(October 8, 1993)

\begin{abstract}
As gravity is a long-range force, one might a priori expect the Universe's global matter distribution to select a preferred rest frame for local gravitational physics. At the post-Newtonian approximation, two parameters suffice to describe the phenomenology of preferred-frame effects. One of them has already been very tightly constrained $\left(\left|\alpha_{2}\right|<4 \times 10^{-7}, 90 \%\right.$ C.L.), but the present bound on the other one is much weaker $\left(\left|\alpha_{1}\right|<5 \times 10^{-4}, 90 \%\right.$ C.L. $)$. It is pointed out that the observation of particular orbits of artificial Earth satellites has the potential of improving the $\alpha_{1}$ limits by a couple of orders of magnitude, thanks to the appearance of small divisors which enhance the corresponding preferred-frame effects. There is a discrete set of inclinations which lead to arbitrarily small divisors, while, among zero-inclination (equatorial) orbits, geostationary ones are near optimal. The main $\alpha_{1}$-induced effects are: (i) a complex secular evolution of the eccentricity vector of the orbit, describable as the vectorial sum of several independent rotations; and (ii) a yearly oscillation in the longitude of the satellite.
\end{abstract}

PACS numbers: 04.80.+z, 95.40.+s, 11.30.Cp 


\section{INTRODUCTION}

The absence of preferred frames in local experiments (or local boost invariance) is verified everyday in high-energy experiments but is much more difficult to test for the gravitational interaction. Metrically coupled tensor-scalar theories of gravity (à la Jordan-Fierz-BransDicke), including general relativity, do not predict any violation of this invariance (see e.g. [1] and references therein). On the other hand, one expects the Universe's global matter distribution to select a preferred rest frame for the gravitational interaction if it is mediated in part by a long-range vector field (or a second tensor field besides the unique one postulated by Einstein) [2]. Tests of the boost-invariance of gravity in localized systems are therefore of special interest to determine the field content of the gravitational interaction, i.e. specifically whether gravity contains, besides the standard tensor contribution and an often considered scalar one, extra contributions due to the exchange of a vector or a second tensor field.

In the post-Newtonian limit, all preferred frame effects are phenomenologically describable by only two parameters, $\alpha_{1}$ and $\alpha_{2}$ [2]. [Note that the post-Newtonian approach assumes that all the fields contributing to gravity are massless, or at least have a range much larger than the size of the considered $N$-body system.] These two preferred-frame parameters contribute non-boost-invariant terms in the Lagrangian, depending on the velocities $\mathbf{v}^{0}$ of the bodies with respect to some gravitationally preferred rest frame. More precisely, the post-Newtonian Lagrangian describing the interaction between $N$ spherical bodies reads

$$
\begin{aligned}
L^{N \text { body }}= & L_{\beta, \gamma, \eta}+L_{\alpha_{1}}+L_{\alpha_{2}}, \\
L_{\beta, \gamma, \eta}= & \sum_{A}-m_{A} c^{2}\left(1-\left(\mathbf{v}_{A}^{0}\right)^{2} / c^{2}\right)^{1 / 2} \\
& +\frac{1}{2} \sum_{A \neq B} \frac{G_{A B} m_{A} m_{B}}{r_{A B}}\left[1+\frac{1}{2 c^{2}}\left(\left(\mathbf{v}_{A}^{0}\right)^{2}+\left(\mathbf{v}_{B}^{0}\right)^{2}\right)-\frac{3}{2 c^{2}}\left(\mathbf{v}_{A}^{0} \cdot \mathbf{v}_{B}^{0}\right)\right. \\
& \left.-\frac{1}{2 c^{2}}\left(\mathbf{n}_{A B} \cdot \mathbf{v}_{A}^{0}\right)\left(\mathbf{n}_{A B} \cdot \mathbf{v}_{B}^{0}\right)+\frac{\gamma}{c^{2}}\left(\mathbf{v}_{A}^{0}-\mathbf{v}_{B}^{0}\right)^{2}\right] \\
& -\frac{1}{2} \sum_{B \neq A \neq C}(2 \beta-1) \frac{G^{2} m_{A} m_{B} m_{C}}{c^{2} r_{A B} r_{A C}}, \\
L_{\alpha_{1}}= & -\frac{\alpha_{1}}{4} \sum_{A \neq B} \frac{G m_{A} m_{B}}{c^{2} r_{A B}}\left(\mathbf{v}_{A}^{0} \cdot \mathbf{v}_{B}^{0}\right), \\
L_{\alpha_{2}}= & \frac{\alpha_{2}}{4} \sum_{A \neq B} \frac{G m_{A} m_{B}}{c^{2} r_{A B}}\left[\left(\mathbf{v}_{A}^{0} \cdot \mathbf{v}_{B}^{0}\right)-\left(\mathbf{n}_{A B} \cdot \mathbf{v}_{A}^{0}\right)\left(\mathbf{n}_{A B} \cdot \mathbf{v}_{B}^{0}\right)\right],
\end{aligned}
$$

where the effective coupling constant $G_{A B}$ for the gravitational interaction of bodies $A$ and $B$ is

$$
G_{A B}=G\left[1+\eta\left(\frac{E_{A}^{\mathrm{grav}}}{m_{A} c^{2}}+\frac{E_{B}^{\mathrm{grav}}}{m_{B} c^{2}}\right)\right],
$$

in which $\eta$ denotes the combination of parameters

$$
\eta \equiv 4 \beta-\gamma-3-\alpha_{1}+\frac{2}{3} \alpha_{2}
$$


while $E_{A}^{\text {grav }} \equiv-(G / 2) \int_{A} \int_{A} d^{3} x d^{3} x^{\prime} \rho(\mathbf{x}) \rho\left(\mathbf{x}^{\prime}\right) /\left|\mathbf{x}-\mathbf{x}^{\prime}\right|$ denotes the gravitational self-energy of body $A$. Apart from the contributions of $\alpha_{1}$ and $\alpha_{2}$ to $G_{A B}$ (via $\eta$ ), $L_{\beta, \gamma, \eta}$ is the usual boost-invariant Lagrangian involving the Eddington parameters $\beta$ and $\gamma$, which are sufficient to parametrize the whole class of metrically-coupled tensor-multi-scalar theories $(\beta=\gamma=$ 1 in general relativity, while these parameters can take arbitrary values in tensor-scalar theories). The parameters $\alpha_{1}$ and $\alpha_{2}$ contribute to the effects associated with a violation of the strong equivalence principle $\left(G_{A B} \neq G\right)$ only through the combined parameter $\eta$. Observational tests of the parameter $\eta$ are discussed in the literature (e.g. [3] ). In the following we concentrate on the effects associated to the velocity-dependent Lagrangian $L_{\alpha_{1}}$ of Eq. (3).

It has been shown in Ref. [4] that the close alignment of the Sun's spin axis with the solar system's planetary angular momentum yields an extremely tight bound on $\alpha_{2}$

$$
\left|\alpha_{2}\right|<3.9 \times 10^{-7} \quad(90 \% \text { C.L. }) .
$$

This limit on $\alpha_{2}$ is much stronger than the existing limits on the other post-Newtonian parameters $\beta, \gamma$ and $\alpha_{1}$ [5]. Present experimental bounds on the Eddington parameters are at the level [3]

$$
|\gamma-1|<3 \times 10^{-3} \quad, \quad|\beta-1|<3 \times 10^{-3} \quad \text { (90\% C.L.) },
$$

while the limits on $\alpha_{1}$ are about six times tighter [3,6 9]. More precisely combined orbital data on the planetary system yield [8]

$$
\alpha_{1}=(2.1 \pm 3.1) \times 10^{-4} \quad(90 \% \text { C.L. }),
$$

while the bound obtained in the strong-field regime by analyzing binary-pulsar data is comparable [9]

$$
\left|\alpha_{1}\right|<5.0 \times 10^{-4} \quad(90 \% \text { C.L. }) .
$$

Recent theoretical developments in tensor-scalar cosmological models 10 suggest that a natural level for $|\beta-1|$ and $|\gamma-1|$ is $\sim 10^{-5}-10^{-7}$. The generalization of this result to classes of gravitational theories involving extra vector or tensor interactions has not been worked out, but, by analogy, one might expect the present limit on $\alpha_{1}$ to be too weak to constrain at a significant level the participation to gravity of extra vector or tensor fields. It is therefore worth discussing experiments having the capability of improving the existing limits on $\alpha_{1}$ down to the $10^{-5}-10^{-6}$ level.

The object of the present paper is to show that artificial Earth satellites offer very promising tools to improve the precision of measurement of $\alpha_{1}$ by, possibly, a couple of orders of magnitude. Indeed, we find that the appearance of small divisors can considerably enhance preferred-frame effects when the semi-major axis of the satellite's orbit and/or its inclination with respect to the Earth's equatorial plane take particular values. Section II is devoted to the secular evolution equations satisfied by the orbital elements, whose $\alpha_{1}$ dependent contributions derive from the disturbing function (13). As a first example of a small divisor which enhances preferred-frame effects, we consider in section III the simple case of equatorial orbits, and show that because of a competition between tidal forces and 
the quadrupolar moment of the Earth, there exists an optimal value of the semi-major axis which maximizes the perturbations due to $\alpha_{1}$. It turns out that geostationary satellites are nearly optimal. The case of non-equatorial orbits is studied in section IV. We point out that preferred-frame effects can be enhanced by arbitrarily large factors if the inclination is sufficiently close to one of six specific values, so that the accuracy of measurement of $\alpha_{1}$ becomes limited only by the finite duration of the experiment.

When deriving bounds on $\alpha_{1}$ or $\alpha_{2}$ [such as Eqs. (7),(9),(10)], it is necessary to make a definite assumption about the preferred rest frame entering the Lagrangians (3),(4). The standard assumption [3, 4, 6-9], that we shall take up in the present paper, is to choose the frame defined by the cosmic microwave background [this essentially means that the range of the putative extra vector or tensor field responsible for the violation of local boost invariance is at least of cosmological magnitude]. However, we shall see in section V.A that (somewhat weaker) bounds on $\alpha_{1}$ can be obtained without the need of such a hypothesis. Indeed, the 'absolute' velocity of an artificial Earth satellite can be decomposed as $\mathbf{v}_{\text {sat }}^{0}=\mathbf{v}_{\text {sat }}+\mathbf{v}_{\oplus}+\mathbf{w}$, where $\mathbf{v}_{\text {sat }}$ is the velocity of the satellite with respect to the Earth, $\mathbf{v}_{\oplus}$ the orbital velocity of the Earth around the Sun, and $\mathbf{w}$ the velocity of the Sun with respect to the gravitationally

preferred frame. The $\alpha_{1}$-contribution (3) to the interaction term between the Earth and the satellite reads then

$$
L_{\alpha_{1}}=-\frac{\alpha_{1}}{2} \frac{G m_{\oplus} m_{\mathrm{sat}}}{c^{2} r_{\oplus \mathrm{sat}}}\left(\mathbf{v}_{\oplus}+\mathbf{w}\right) \cdot\left(\mathbf{v}_{\mathrm{sat}}+\mathbf{v}_{\oplus}+\mathbf{w}\right) .
$$

This involves a term proportional to $\mathbf{v}_{\oplus} \cdot \mathbf{v}_{\text {sat }}$ which leads to observable effects, although they are 12 times smaller than those proportional to $\mathbf{w} \cdot \mathbf{v}_{\text {sat }}$, since $v_{\oplus} / c \approx 9.94 \times 10^{-5}$ whereas $w / c \approx 1.22 \times 10^{-3}$ [1]. Section V.B is devoted to the effects involving $\alpha_{1} v_{\oplus}$ that are neglected in the rest of the paper. In particular, we show there that the term proportional to $\mathbf{w} \cdot \mathbf{v}_{\oplus}$ in Eq. (11) leads to sizable deviations of the angular position of the satellite, which could be used to constrain the value of $\alpha_{1}$ independently of the tests proposed in sections III and IV. We finally summarize our results and give our conclusions in section VI.

\section{SECULAR VARIATIONS OF THE ORBITAL ELEMENTS}

The preferred-frame effects described by the Lagrangians (3) and (4) are $\sim \alpha_{1} v^{2} / c^{2}$ smaller than the leading Newtonian interaction, and lead, over one orbital period, to very small deviations of the position of a satellite. Taking into account the present limits (9)-(10), one expects $\alpha_{1}$-induced displacements $|\delta \mathbf{x}| \sim \alpha_{1} w v_{\text {sat }} r_{\oplus \text { sat }} / 2 c^{2} \lesssim 75 \mu \mathrm{m}$ (for $r_{\oplus \text { sat }} \sim 2 R_{\oplus}$ ), too small to be observable with present or foreseeable techniques. Fortunately, some of these perturbations build up beyond one orbital period, and can thus be enhanced to an observable level if one waits for a sufficiently large number of periods. We restrict our attention to such effects in this paper, i.e. we concentrate on secular variations of the orbital elements. Concentrating on secular effects has also the advantage of freeing us from the coordinate ambiguities present in orbital-period effects [12].

When taking into account only the Newtonian potential generated by a spherical Earth $G m_{\oplus} m_{\text {sat }} / r_{\oplus \text { sat }}$, the satellite's orbit is determined by six constants of motion: its semimajor axis $a$, its eccentricity $e$, its inclination $I$ with respect to the Earth's equatorial 
plane, the angle $\Omega$ between a direction of reference $(\alpha, \delta)=(0,0)$ [13] and the ascending node, the angle $\omega$ between this ascending node and the perigee, and finally the quantity $\sigma$ entering the mean anomaly $\ell \equiv \int_{0}^{t} n\left(t^{\prime}\right) d t^{\prime}+\sigma$, where $n \equiv 2 \pi / P=\left(G m_{\oplus} / a^{3}\right)^{1 / 2}$ is the orbital frequency. [Numerically $P=\left(a / R_{\oplus}\right)^{3 / 2} \times 1.406 \mathrm{~h}$, when using $R_{\oplus}=6.371 \times 10^{6} \mathrm{~m}$, $G m_{\oplus}=3.986 \times 10^{14} \mathrm{~m}^{3} \mathrm{~s}^{-2}$.] To help visualizing the meaning of the angle $\sigma$, it is useful to note that, in the limiting case of a small eccentricity $e$, the mean anomaly $\ell$ can be identified with the angular position of the satellite, so that $\sigma$ can be viewed as the angle between the perigee and the satellite at $t=0$. Figure 1 summarizes this notation. It will also be useful in the following to define an orthonormal triad $(\mathbf{a}, \mathbf{b}, \mathbf{c})$, where $\mathbf{a}$ is directed towards the ascending node and $\mathbf{c}=\mathbf{a} \times \mathbf{b}$ is in the direction of the orbital angular momentum, i.e. orthogonal to the orbital plane. (Note that this triad is not the one used in references [9, 14], where a was directed towards the periastron.)

We find it convenient to use the method of variation of the elements, as described for instance in Ref. [15], to derive the secular variations of $a, e, I, \sigma, \omega$ and $\Omega$. For more generality, let us consider a perturbed two-body system $\left(m_{A}, m_{B}\right)$, define $M \equiv m_{A}+m_{B}$, $X_{A} \equiv m_{A} / M, X_{B} \equiv m_{B} / M$, and write the Lagrangian as $L=L_{0}+M X_{A} X_{B} R$ where $L_{0}$ contains, besides the kinetic terms, only the Newtonian potential between spherical bodies $G m_{A} m_{B} / r_{A B}$, and $R$ is the disturbing function containing all corrective terms due to asphericities, tidal forces, relativistic effects, ... We shall not consider here the motion of the center of mass of the two-body system [16], but concentrate upon the equations satisfied by the elements of the relative orbit $\mathbf{x}_{A}-\mathbf{x}_{B}$. These derive directly from $R$, and read [15]

$$
\begin{aligned}
\frac{d a}{d t} & =\frac{2}{n a} \frac{\partial R}{\partial \sigma} \\
\frac{d e}{d t} & =\frac{1-e^{2}}{n a^{2} e} \frac{\partial R}{\partial \sigma}-\frac{\left(1-e^{2}\right)^{1 / 2}}{n a^{2} e} \frac{\partial R}{\partial \omega}, \\
\frac{d I}{d t} & =\frac{\cot I}{n a^{2}\left(1-e^{2}\right)^{1 / 2}} \frac{\partial R}{\partial \omega}-\frac{1}{n a^{2}\left(1-e^{2}\right)^{1 / 2} \sin I} \frac{\partial R}{\partial \Omega}, \\
\frac{d \sigma}{d t} & =-\frac{2}{n a}\left[\frac{\partial R}{\partial a}\right]-\frac{1-e^{2}}{n a^{2} e} \frac{\partial R}{\partial e}, \\
\frac{d \omega}{d t} & =\frac{\left(1-e^{2}\right)^{1 / 2}}{n a^{2} e} \frac{\partial R}{\partial e}-\frac{\cot I}{n a^{2}\left(1-e^{2}\right)^{1 / 2}} \frac{\partial R}{\partial I}, \\
\frac{d \Omega}{d t} & =\frac{1}{n a^{2}\left(1-e^{2}\right)^{1 / 2} \sin I} \frac{\partial R}{\partial I}
\end{aligned}
$$

The brackets in the right hand side of Eq. (12di) indicate that the $a$-differentiation is effected keeping the mean anomaly fixed (i.e. ignoring the implicit $a$-dependence contained in $\int n d t$ ). When working to first order in $R$, the orbital elements can be replaced by their constant zeroth order values on the right-hand-sides. Then the secular variations of the orbital elements are simply obtained by replacing in equations (12) $R$ by its average value $\langle R\rangle$, computed over one unperturbed Newtonian orbit [More precisely, one takes the average over the angle $\ell$, keeping the other elements fixed]. It is straightforward to integrate the terms proportional to $1 / c^{2}$ in the two-body versions of the Lagrangians (2) and (3). Decomposing the 'absolute' velocities as $\mathbf{v}_{A, B}^{0}=\mathbf{v}_{A, B}+\mathbf{w}_{\mathrm{CM}}$, where $\mathbf{w}_{\mathrm{CM}}$ is the velocity of the 
center-of-mass of the system $(A, B)$ with respect to the preferred rest frame, we find the contribution

$$
\begin{aligned}
\left\langle R_{\alpha_{1}, \beta, \gamma}\right\rangle= & -\frac{\alpha_{1}}{2}\left(\frac{G M}{a c^{2}}\right) c^{2}\left(\frac{w_{\mathrm{CM}}}{c}\right)^{2} \\
& +\frac{\alpha_{1}}{2}\left(\frac{G M}{a c^{2}}\right)^{3 / 2} c^{2}\left(X_{A}-X_{B}\right) \frac{\left[\mathbf{w}_{\mathrm{CM}} / c, \mathbf{c}, \mathbf{e}\right]}{1+\left(1-e^{2}\right)^{1 / 2}} \\
& +\left(\frac{G M}{a c^{2}}\right)^{2} c^{2}\left(\frac{2 \gamma-\beta+2+\alpha_{1} X_{A} X_{B}}{\left(1-e^{2}\right)^{1 / 2}}-\frac{8 \gamma+7-X_{A} X_{B}\left(1-4 \alpha_{1}\right)}{8}\right),
\end{aligned}
$$

where the square brackets denote the mixed product $\left(\mathbf{w}_{\mathrm{CM}} / c\right) \cdot(\mathbf{c} \times \mathbf{e})$, the vector $\mathbf{e}$ being the Lagrange-Laplace (-Runge-Lenz) eccentricity vector with norm $e$, directed towards the periastron of body $A$. The first line comes from the term proportional to $w_{\mathrm{CM}}^{2}$ in $L_{\alpha_{1}}$ (3), and the second from the term involving $\mathbf{w}_{\mathrm{CM}} \cdot\left(\mathbf{v}_{A}+\mathbf{v}_{B}\right)$. The third line is responsible for the usual relativistic perigee advance and comes from the $1 / c^{2}$ terms of the Lagrangian (1), with extra contributions due to the $\mathbf{v}_{A} \cdot \mathbf{v}_{B}$ term of $L_{\alpha_{1}}$. We have not included in Eq. (13) the contribution of $\alpha_{2}$, relying on the limit (7) to consider that this type of preferred effects is already plausibly excluded.

Up to here, we have considered a two-body system with arbitrary masses $\left(m_{A}, m_{B}\right)$ for generality's sake. In the particular case of artificial Earth satellites, one can however neglect $m_{\text {sat }}$ with respect to $m_{\oplus}$, and therefore write $M \approx m_{\oplus}, X_{A}=X_{\text {sat }} \approx 0, X_{B}=X_{\oplus} \approx 1$. Hence, we see from the last line in Eq. (13) that the additional contribution to the relativistic perigee advance generated by $\alpha_{1}$ is completely negligible. As for the first term on the right hand side of Eq. (13), it only contributes to the evolution of the element $\sigma$ and will be studied in section V.B. In the next two sections we concentrate on the evolution of the other elements, generated by the second term on the r.h.s. of Eq. (13).

The contribution (13) should be added to the usual Newtonian multipolar and tidal perturbations, notably the effect of the Earth's quadrupolar moment $\left(J_{2}\right)$ and the tidal forces due to the Moon (denoted as $\mathbb{C}$ ) and the Sun (denoted as $\odot$ ), which are the dominant ones. For analytical simplicity, we shall not consider any other Newtonian perturbation in the present paper. Our estimates of the measurability of $\alpha_{1}$ will correspondingly be only indicative. To get reliable estimates of the bounds on $\alpha_{1}$ which could be experimentally obtained, it would be necessary to perform detailed numerical simulations taking into account all known Newtonian and general relativistic effects, as well as all modelizable sources of noise. [There exist in particular resonances between several multipolar moments of the Earth which have significant effects on long times.]

The secular contribution due to the Earth's oblateness can be written down very easily

$$
\left\langle R_{J_{2}}\right\rangle=J_{2} \frac{G m_{\oplus} R_{\oplus}^{2}}{a^{3}} \frac{2-3 \sin ^{2} I}{4\left(1-e^{2}\right)^{3 / 2}} .
$$

The contribution of tidal forces is more complicated (even if one neglects the eccentricities of the Earth's and the Moon's orbits, as well as their inclinations with respect to the Earth's equatorial plane) because it involves explicitly the angular positions of the Moon and the Sun. However, it can be simplified very much if the variations of the orbital elements (12) 
are studied on longer time scales, more precisely if one averages over the orbital periods of the Moon around the Earth ( one month) and of the Earth around the Sun (one year). The variations of the satellite's orbital elements after such averagings can then be derived from

$$
\left\langle\left\langle R_{\text {Tides }}\right\rangle\right\rangle=N^{2} \frac{a^{2}}{8}\left[2+3 e^{2}-3 \sin ^{2} I\left(1-e^{2}+5 e^{2} \sin ^{2} \omega\right)\right]
$$

where

$$
N^{2} \equiv \frac{G m_{\mathbb{C}}}{r_{\oplus \mathbb{C}}^{3}}+\frac{G m_{\odot}}{r_{\oplus \odot}^{3}} .
$$

[The notation $N$ is reminiscent of the fact that $n_{\oplus} \equiv\left(G m_{\odot} / r_{\oplus \odot}^{3}\right)^{1 / 2}$ is the orbital frequency of the Earth around the Sun; note however that $\left(G m_{\mathbb{C}} / r_{\oplus \mathbb{C}}^{3}\right)^{1 / 2}$ is not the orbital frequency of the Moon around the Earth, which would involve $m_{\oplus}+m_{\mathbb{C}}$ instead of $m_{\mathbb{C}}$.] Beware that the above expression (15) should not be used to derive variations with periods $\lesssim$ one year.

The secular variations of the satellite's orbital elements can now be easily derived from Eqs. (12). They tell us that the semi-major axis undergoes no secular variation $(\langle\dot{a}\rangle=$ $0)$, and that the change of the inclination is negligible in the limit of a small eccentricity $\left[\langle d I / d t\rangle=O\left(\alpha_{1} e\right)+O\left(e^{2}\right)\right]$. We shall study the equations satisfied by $e, \omega$ and $\Omega$ in sections III and IV, and see that they can be rewritten more compactly as a vectorial equation for the eccentricity vector $\mathbf{e}$ in the limit $e \ll 1$. Section V.B will be devoted to the equation satisfied by $\sigma$.

Finally, it should be noted that in the main body of the paper we always deal with the motion of a satellite as seen in a (locally inertial) geocentric frame. The $\alpha_{1}$-dependent effects linked to the connection between such a geocentric frame and a global, barycentric one will be briefly discussed in the concluding section.

\section{COMPETITION BETWEEN TIDAL FORCES AND EARTH'S OBLATENESS FOR EQUATORIAL ORBITS}

The aim of this section is to show on the simple example of equatorial orbits how small divisors can enhance the preferred-frame effects associated to $\alpha_{1}$. [As said above, we neglect the eccentricities of the Earth's and the Moon's orbits, as well as their inclinations with respect to the Earth's equatorial plane.] The secular variations of the satellite's orbital elements are then given by equations (12), where $\langle R\rangle$ is the sum of Eqs. (13), (14) and (15) with $I=0$. However, the angular position of the ascending node is no longer well-defined as $I \rightarrow 0$, and equations $(12 \mathrm{e})$ and $(\overline{12 \mathrm{f}})$ become formally singular. As is well known [15], this singularity is fictitious and taken care of by considering the evolution of the angle $\widetilde{\omega} \equiv \omega+\Omega$ between the direction of reference $(\alpha, \delta)=(0,0)$ and the perigee, which stays well-defined in the limit $I \rightarrow 0$. One finds

$$
\begin{aligned}
\left\langle\frac{d \widetilde{\omega}}{d t}\right\rangle & =\frac{\left(1-e^{2}\right)^{1 / 2}}{n a^{2} e} \frac{\partial\langle R\rangle}{\partial e} \\
& =\dot{\widetilde{\omega}}_{N}-\frac{\alpha_{1}}{4} \frac{n^{2} a}{c} \frac{\left[\mathbf{w}_{\oplus} / c, \mathbf{c}, \mathbf{e}\right]}{e^{2}}+O\left(\alpha_{1} e\right)
\end{aligned}
$$


where

$$
\dot{\widetilde{\omega}}_{N} \equiv n\left[\frac{3}{2} J_{2}\left(\frac{R_{\oplus}}{a}\right)^{2}+\frac{3}{4} \frac{N^{2}}{n^{2}}+O\left(e^{2}\right)\right]
$$

is the Newtonian perigee advance due to the Earth's quadrupolar moment and tidal forces, and $\mathbf{w}_{\oplus} \equiv \mathbf{w}+\mathbf{v}_{\oplus}$ the velocity of the Earth with respect to the preferred frame, $\mathbf{v}_{\oplus}$ denoting as in section I the orbital velocity of the Earth around the Sun. [It is useful to quote here the numerical value of $\frac{3}{2} n J_{2}\left(R_{\oplus} / a\right)^{2}=\left(R_{\oplus} / a\right)^{7 / 2} \times 2.02 \times 10^{-6} \mathrm{~s}^{-1} \approx\left(R_{\oplus} / a\right)^{7 / 2} \times 2 \pi /(0.10 \mathrm{yr})$, which will appear again in the following sections as the characteristic frequency for the Newtonian variations of the orbital elements $\omega$ and $\Omega$; see notably Eqs. (29) and (31).] Equation (17) together with the one satisfied by $e$

$$
\left\langle\frac{d e}{d t}\right\rangle=-\frac{\alpha_{1}}{4} \frac{n^{2} a}{c}\left(\frac{\mathbf{w}_{\oplus}}{c} \cdot \frac{\mathbf{e}}{e}\right)+O\left(\alpha_{1} e^{2}\right)
$$

can be rewritten as a simple vectorial equation for the eccentricity vector $\mathbf{e}$ in the limit $e \ll 1$

$$
\left\langle\frac{d \mathbf{e}}{d t}\right\rangle=\mathbf{c} \times\left[\dot{\widetilde{\omega}}_{N} \mathbf{e}+\left(\mathbf{k}+\boldsymbol{\kappa}_{\oplus}\right) \times \mathbf{c}\right]+O\left(\alpha_{1} e^{2}\right)+O\left(e^{2}\right)
$$

where

$$
\mathbf{k} \equiv-\alpha_{1} \frac{G m_{\oplus}}{4 a^{2} c^{2}} \mathbf{w}
$$

is a constant vector (beware that Ref. [9] defines a vector $\mathbf{k}$ which is equal to twice this value) and

$$
\boldsymbol{\kappa}_{\oplus}(t) \equiv-\alpha_{1} \frac{G m_{\oplus}}{4 a^{2} c^{2}} \mathbf{v}_{\oplus}
$$

a yearly varying one. In the present and following sections, we concentrate on the effects of the constant vector $\mathbf{k}$, leaving to section V.A a study of the effects associated to $\boldsymbol{\kappa}_{\oplus}(t)$. Since we are considering equatorial orbits in this section, the vector $\mathbf{c}$ orthogonal to the orbital plane is the constant unitary vector parallel to the Earth's polar axis, and therefore $\mathbf{c} \times(\mathbf{k} \times \mathbf{c})$ is a constant vector, namely the projection of $\mathbf{k}$ onto the equatorial plane. Hence a $\alpha_{1}$-type violation of local boost invariance has the consequence of adding a constant forcing term in the time evolution of the eccentricity vector which, if it were alone, would secularly 'polarize' the orbit in the direction of the equatorial projection of $\mathbf{w}$. However, the Newtonian precession term $\dot{\widetilde{\omega}}_{N}$ cuts off the build up of this polarizing term and deflects it by $90^{\circ}$ in a gyroscope-like way. More precisely, the solution of Eq. (20) can be written as the vectorial superposition

$$
\mathbf{e}=\mathbf{e}_{N}(t)+\mathbf{e}_{F}
$$

where $\mathbf{e}_{N}(t)$ is a vector of constant norm rotating with angular frequency $\dot{\widetilde{\omega}}_{N}$ in the orbital plane (usual Newtonian perigee advance), and $\mathbf{e}_{F}$ is the fixed polarizing contribution due to the preferred-frame effects we are studying, namely 


$$
\mathbf{e}_{F} \equiv \frac{\mathbf{c} \times \mathbf{k}}{\dot{\tilde{\omega}}_{N}}
$$

Note the factor $\left(\dot{\widetilde{\omega}}_{N}\right)^{-1}$ in Eq. (24) which is the first appearance of a small divisor. The solution (23) is formally identical to the one found for binary pulsars in Ref. [9], the only difference being that $\dot{\tilde{\omega}}_{N}$ replaces the relativistic periastron advance. The same kind of polarizing terms in the time evolution of e has also been pointed out previously in [14], in the totally different context of equivalence principle violation in binary systems.

In geometrical terms, Eq. (23) means that the eccentricity vector $\mathbf{e}(t)$ traces out, during its time evolution (after averaging over an orbital period), a circle centered around $\mathbf{e}_{F}$. If a large enough segment of this circle (say about a quarter) can be monitored during the experiment, it should be possible to measure the position of its center, i.e. $\mathbf{e}_{F}$, with about the same precision that individual measurements of the eccentricity vector [17]. In turn, the precision of the measurement of $\mathbf{e}$ is related to the precision $\sigma_{\mathbf{x}}$ with which one can measure the satellite's position: roughly one expects $\sigma_{\mathbf{e}} \approx \sigma_{\mathbf{x}} / a$. It is convenient to work with quantities homogeneous to displacements. Therefore we shall measure the $\alpha_{1}$-perturbations of $\mathbf{e}$ in terms of

$$
\delta \rho=a e_{F}=\frac{\alpha_{1}}{3 c^{2}} \frac{|\mathbf{c} \times \mathbf{w}|\left(G m_{\oplus}\right)^{3 / 2}}{2 J_{2} G m_{\oplus} R_{\oplus}^{2} a^{-5 / 2}+N^{2} a^{5 / 2}},
$$

which represents (when $e_{F}<e_{N}$, as expected from $e_{F} \leq \alpha_{1} \times 5.7 \times 10^{-5}<3 \times 10^{-8}$ ) the amplitude of the secular change in the distance to the perigee $\rho=a(1-|\mathbf{e}|)$. From the arguments just given, equating $\delta \rho$ with the position measurement precision $\sigma_{\mathbf{x}}$ should yield an estimate of the precision with which $\alpha_{1}$ can be measured over a time span $T / 4$, where

$$
T \equiv \frac{2 \pi}{\dot{\widetilde{\omega}}_{N}}
$$

The denominator of the expression (25) is a function of the semi-major axis $a$, and is minimized for the value

$$
\begin{aligned}
a_{\text {optimal }} & =\left(2 J_{2} G m_{\oplus} R_{\oplus}^{2} / N^{2}\right)^{1 / 5} \\
& \approx 7.66 R_{\oplus} \approx 4.88 \times 10^{7} \mathrm{~m}
\end{aligned}
$$

(which corresponds to an orbital period $\sim 30$ hours). In Eq. (27), we have used the numerical data $J_{2}=1.08263 \times 10^{-3}, G m_{\oplus} / R_{\oplus} c^{2}=6.97 \times 10^{-10}, R_{\oplus}=6.371 \times 10^{6} \mathrm{~m}$, and $N=3.56 \times 10^{-7} \mathrm{~s}^{-1}$. The maximal value of $\delta \rho$ (for equatorial orbits) is thus obtained for this value of the semi-major axis, and reads

$$
\begin{aligned}
\delta \rho_{\max } & =\alpha_{1} \frac{G m_{\oplus}}{R_{\oplus} c^{2}} \frac{c}{6 N\left(2 J_{2}\right)^{1 / 2}} \frac{w_{\text {equatorial }}}{c} \\
& \approx \alpha_{1} \times 2.54 \times 10^{5} \mathrm{~cm}
\end{aligned}
$$

where the norm $w / c=1.22 \times 10^{-3}$ and the direction $(\alpha, \delta)=\left(11.2 \mathrm{~h},-7^{\circ}\right)$ of $\mathbf{w}$ have been extracted from the results of COBE 11 . 
Present technologies make it possible to measure the position of a satellite down to $\sigma_{\mathbf{x}} \sim$ $1 \mathrm{~cm}$, by using either laser ranging (as for the Laser Geodynamical Satellites 'LAGEOS') or Global Positioning System (GPS) receivers onboard. The displacement (28) could therefore lead in principle to limits on $\alpha_{1}$ of the order of $4 \times 10^{-6}$, i.e. two orders of magnitude tighter than the present bounds (9) and (10). It is to be noted that a geostationary satellite $\left(a \approx 6.62 R_{\oplus}\right.$, period $\left.\approx 23 \mathrm{~h} 56 \mathrm{~min}\right)$ is near optimal. It yields $\delta \rho \approx \alpha_{1} \times 2.4 \times 10^{5} \mathrm{~cm}$, leading us to expect a precision $\alpha_{1} \sim 4.2 \times 10^{-6}$. Clearly, numerical simulations taking into account all known Newtonian perturbations and modelizable non-conservative forces should be performed in order to get more realistic estimates of the precision which can be reached.

This simple case of equatorial orbits already exhibits one of the characteristic behaviors of preferred-frame effects enhanced by small divisors: the duration of the experiment must be large enough to take advantage of the small divisor effect. For instance, the time span of the experiment should be (at least) $T / 4=\pi / 2 \dot{\widetilde{\omega}}_{N} \sim 15.4$ yr in the case of the optimal orbit (27), and $\sim 12.4$ yr for a geostationary satellite.

Let us conclude this section by a comparison of the result (28) with the one corresponding to the best drag-free satellite we know: the Moon itself. Still neglecting the inclination of its orbit and that of the ecliptic with respect to the Earth's equatorial plane, we can repeat all of the above discussion, except that in Eq. (16) only the tidal forces due to the Sun should be taken into account. Actually, higher-order terms in these tidal forces are not small in the particular case of the Moon (because its orbital period is non-negligible as compared to one year), and the coefficient $N^{2}$ of Eq. (15) should be replaced by $2.043 n_{\oplus}^{2}$ instead of merely $n_{\oplus}^{2} \equiv G m_{\odot} / r_{\oplus \odot}^{3}$ to yield the correct magnitude of $\dot{\widetilde{\omega}}_{N}$ [15]. The amplitude of the secular oscillation of the Moon's perigee distance is then given by the same formula (25) as above with $a=r_{\oplus \mathbb{C}}=60.3 R_{\oplus}$. The Earth's oblateness gives then a negligible contribution to the denominator of $\delta \rho$ in Eq. (25), and one finds $\delta \rho_{\mathbb{C}} \approx \alpha_{1} \times 4.5 \times 10^{3} \mathrm{~cm}$, with period $T \approx 8.72 \mathrm{yr}$. This effect was first discussed (in a different guise) in Ref. [18]. Since Lunar Laser Ranging (LLR) allows one to determine the Earth-Moon distance within 2 or 3 centimeters, we expect that an analysis of LLR data could at best limit $\alpha_{1}$ at the $\sim 5 \times 10^{-4}$ level, i.e. not better than the existing bounds (9),(10). However, as this would represent a new, independent test of preferred-frame effects, it would be interesting to perform explicitly a multi-parameter fit of LLR data including the contributions of $\alpha_{1}$ (and for completeness $\alpha_{2}, \beta, \gamma$ ) to the Lunar equations of motion.

\section{NON-EQUATORIAL ORBITS AND ARBITRARILY SMALL DIVISORS}

In this section, we shall see that non-equatorial orbits (of any altitude) can give rise to arbitrarily small divisors if the inclination is near some special values. For reasons that will appear clear below, we concentrate upon rather low satellites, for which tidal forces are negligible compared to the influence of the Earth's quadrupolar moment. For this reason we do not take into account the disturbing function (15) in the present section, in order to avoid unnecessarily technical calculations. However, we show at the end of this section how the equations satisfied by the orbital elements can be solved when tidal forces are not neglected.

The main difference between equatorial and non-equatorial satellites is that the orbital 
plane is no longer fixed when $I \neq 0$. Indeed, equation (12f) shows that it is precessing with a constant angular frequency

$$
\langle\dot{\Omega}\rangle=-\frac{3}{2} n J_{2}\left(\frac{R_{\oplus}}{a}\right)^{2} \cos I+O\left(\alpha_{1} e\right)+O\left(e^{2}\right)+O\left(N^{2}\right)
$$

which is not modified by a $\alpha_{1}$-type violation of local Lorentz invariance in the limiting case of a small eccentricity. We shall drop the angular brackets in the following, and denote this constant precessing velocity simply by $\dot{\Omega}$. Like in the previous section, the equations (12b) and (12e) satisfied by $e$ and $\omega$ can be rewritten as a simple vectorial equation for the evolution of the components of $\mathbf{e}$ with respect to the vectors $(\mathbf{a}, \mathbf{b})$, which are part of a frame [defined at the beginning of section II and in Fig. 1] which rotates around the Earth's polar axis with the angular velocity $(\overline{29})$ :

$$
\left\langle d^{\prime} \mathbf{e} / d t\right\rangle=\mathbf{c} \times\left[\dot{\omega}_{N} \mathbf{e}+\left(\mathbf{k}+\boldsymbol{\kappa}_{\oplus}\right) \times \mathbf{c}\right]+O\left(\alpha_{1} e^{2}\right)+O\left(e^{2}\right)+O\left(e N^{2}\right)
$$

where the prime in $d^{\prime} / d t$ denotes a time derivative in the rotating frame $(\mathbf{a}, \mathbf{b}, \mathbf{c})$ and where

$$
\dot{\omega}_{N} \equiv \frac{3}{4} n J_{2}\left(\frac{R_{\oplus}}{a}\right)^{2}\left(4-5 \sin ^{2} I\right)+O\left(e^{2}\right)+O\left(N^{2}\right)
$$

is the Newtonian perigee advance due to the Earth's quadrupolar moment, $\mathbf{k}$ and $\boldsymbol{\kappa}_{\oplus}$ being the vectors defined in Eqs. (21), (22). As in section III, we shall neglect in this section $\boldsymbol{\kappa}_{\oplus}$ with respect to $\mathbf{k}$. Since the projection $\mathbf{c} \times(\mathbf{k} \times \mathbf{c})$ of $\mathbf{k}$ onto the orbital plane is no longer a constant vector because of the precession $\dot{\Omega}$, the solution (23) of the previous section is not valid for non-equatorial orbits. However, it is easy to solve equation (30) which is just an inhomogeneous linear differential equation in e (in the limiting case of a small eccentricity). Let us look for a solution of the type $\mathbf{e}=\mathbf{e}_{N}+\sum_{i} \mathbf{e}_{i}$, where $\mathbf{e}_{N}$ is the usual (constant-norm) Newtonian eccentricity vector rotating in the orbital plane $(\mathbf{a}, \mathbf{b})$ with angular frequency $\dot{\omega}_{N}$, and where the $\mathbf{e}_{i}$ 's are some constant-norm vectors rotating in the orbital plane with constant angular frequencies $\dot{\omega}_{i}$ to be determined. The time derivative of e then reads

$$
\begin{aligned}
d^{\prime} \mathbf{e} / d t & =\mathbf{c} \times\left(\dot{\omega}_{N} \mathbf{e}_{N}+\sum_{i} \dot{\omega}_{i} \mathbf{e}_{i}\right) \\
& =\mathbf{c} \times\left(\dot{\omega}_{N} \mathbf{e}+\sum_{i}\left(\dot{\omega}_{i}-\dot{\omega}_{N}\right) \mathbf{e}_{i}\right) .
\end{aligned}
$$

This has precisely the form of equation (30), with $\mathbf{k} \times \mathbf{c}$ having been decomposed as a sum of constant-norm vectors $\left(\dot{\omega}_{i}-\dot{\omega}_{N}\right) \mathbf{e}_{i}$ rotating with constant angular frequencies $\dot{\omega}_{i}$ in the orbital plane. Since $\mathbf{k} \times \mathbf{c}$ has a periodic motion in the orbital plane (with period $2 \pi / \dot{\Omega}$ ), it obviously admits such a decomposition:

$$
\mathbf{k} \times \mathbf{c}=-\alpha_{1} \frac{G m_{\oplus}}{4 a^{2} c^{2}} w\left(\mathbf{K}_{+}+\mathbf{K}_{0}+\mathbf{K}_{-}\right),
$$

where 


$$
\begin{aligned}
\mathbf{K}_{ \pm} & \equiv \cos \delta \frac{1 \pm \cos I}{2}[\mp \sin (\Omega-\alpha) \mathbf{a}-\cos (\Omega-\alpha) \mathbf{b}] \\
\mathbf{K}_{0} & \equiv \sin \delta \sin I \mathbf{a}
\end{aligned}
$$

$(\alpha, \delta)$ denoting the right-ascension and declination of $\mathbf{w} . \mathbf{K}_{ \pm}$are rotating with angular frequencies $\mp \dot{\Omega}$ in the orbital plane, whereas $\mathbf{K}_{0}$ is a constant vector in this plane (directed towards the ascending node). The solution of Eq. (30) can therefore be written simply as

$$
\begin{aligned}
\mathbf{e} & =\mathbf{e}_{N}+\mathbf{e}_{+}+\mathbf{e}_{0}+\mathbf{e}_{-} \\
& =\mathbf{e}_{N}+\alpha_{1} \frac{G m_{\oplus}}{4 a^{2} c^{2}} w\left(\frac{\mathbf{K}_{+}}{\dot{\omega}_{N}+\dot{\Omega}}+\frac{\mathbf{K}_{0}}{\dot{\omega}_{N}}+\frac{\mathbf{K}_{-}}{\dot{\omega}_{N}-\dot{\Omega}}\right) .
\end{aligned}
$$

This generalizes the solution (23) obtained above for equatorial orbits. [In the limit $I \rightarrow 0$, the term involving $\mathbf{K}_{+}$becomes equal to the vector $\mathbf{e}_{F}$ of Eq. (24), in which $\dot{\omega}_{N}+\dot{\Omega}$ was denoted $\dot{\widetilde{\omega}}_{N}$.] In geometrical terms, Eq. (35) means that the eccentricity vector e $(t)$ undergoes a kind of epicyclic motion in the (a, b) plane: it moves (with angular velocity $\dot{\omega}_{N}$ ) along a circle of radius $\left|\mathbf{e}_{N}\right|$ whose center moves itself (with a non-uniform angular velocity determined by $\dot{\Omega})$ on an ellipse $\left(\mathbf{e}_{+}(t)+\mathbf{e}_{-}(t)\right)$ centered around $\mathbf{e}_{0}$. Hence we can now distinguish three different contributions to the $\alpha_{1}$-induced secular oscillation of the distance to the perigee:

$$
\begin{aligned}
\delta \rho_{ \pm} & =a e_{ \pm}=\alpha_{1} \frac{G m_{\oplus}}{4 a c^{2}} w \cos \delta \frac{(1 \pm \cos I) / 2}{\dot{\omega}_{N} \pm \dot{\Omega}} \\
& =A \cos \delta \frac{(1 \pm \cos I) / 2}{4-5 \sin ^{2} I \mp 2 \cos I}, \\
\delta \rho_{0} & =a e_{0}=\alpha_{1} \frac{G m_{\oplus}}{4 a c^{2}} w \sin \delta \frac{\sin I}{\dot{\omega}_{N}} \\
& =A \sin \delta \frac{\sin I}{4-5 \sin ^{2} I},
\end{aligned}
$$

where

$$
\begin{aligned}
A & \equiv \alpha_{1}\left(\frac{a}{R_{\oplus}}\right)^{5 / 2}\left(\frac{G m_{\oplus}}{R_{\oplus} c^{2}}\right)^{1 / 2} \frac{R_{\oplus}}{3 J_{2}} \frac{w}{c} \\
& \approx \alpha_{1}\left(a / R_{\oplus}\right)^{5 / 2} \times 6316 \mathrm{~cm} .
\end{aligned}
$$

Figure 2 displays the amplitude of these displacements as functions of the inclination. They clearly exhibit poles (or 'resonances') for six particular values of $I: \delta \rho_{+}$diverges for $\dot{\omega}_{N}+\dot{\Omega}=$ 0, i.e. for $I=46.38^{\circ}$ or $106.85^{\circ} ; \delta \rho_{0}$ diverges for $\dot{\omega}_{N}=0$, i.e. for $I=\sin ^{-1}(2 / \sqrt{5})=63.43^{\circ}$ or $116.57^{\circ}$; and $\delta \rho_{-}$diverges for $\dot{\omega}_{N}-\dot{\Omega}=0$, i.e. for $I=73.15^{\circ}$ or $133.62^{\circ}$. (Note that the special value $I_{0} \equiv \sin ^{-1}(2 / \sqrt{5})$ coincides with the well-known Newtonian critical inclination for $J_{2}$-effects [15].) As in the equatorial case discussed above, the price to pay for taking advantage of the small divisors arising near these poles is the need for a long observation period, inversely proportional to the corresponding small divisor. Indeed, the dephasing periods between the Newtonian eccentricity vector $\mathbf{e}_{N}$ and the contributions $\mathbf{e}_{ \pm}, \mathbf{e}_{0}$ proportional to $\alpha_{1}$ read 


$$
\begin{aligned}
T_{ \pm} & =\frac{2 \pi}{\dot{\omega}_{N} \pm \dot{\Omega}}=\frac{B}{4-5 \sin ^{2} I \mp 2 \cos I} \\
T_{0} & =\frac{2 \pi}{\dot{\omega}_{N}}=\frac{B}{4-5 \sin ^{2} I}
\end{aligned}
$$

where

$$
\begin{aligned}
B & \equiv\left(\frac{a}{R_{\oplus}}\right)^{7 / 2}\left(\frac{G m_{\oplus}}{R_{\oplus} c^{2}}\right)^{-1 / 2} \frac{8 \pi}{3 J_{2}} \frac{R_{\oplus}}{c} \\
& \approx\left(a / R_{\oplus}\right)^{7 / 2} \times 0.1974 \mathrm{yr} .
\end{aligned}
$$

[Near each resonance $\dot{\omega}_{N} \pm \dot{\Omega}=0$, one of the vectors $\mathbf{e}_{ \pm}$becomes infinitely large with respect to the other, and the $\mathbf{e}_{+}+\mathbf{e}_{-}$ellipse degenerates to a circle described with angular velocity $\left.\mp \dot{\Omega} \approx \dot{\omega}_{N} \cdot\right]$

In order to compare the merits of the different resonances, it is useful to define as a figure of merit the ratio of the corresponding perigee displacement $\delta \rho$ by the typical (minimal) time $T / 4$ needed for the observation:

$$
\begin{aligned}
\frac{4 \delta \rho_{ \pm}}{T_{ \pm}} & =4 \frac{A}{B} \cos \delta \frac{1 \pm \cos I}{2} \\
& =\alpha_{1} \frac{R_{\oplus}}{a} \times 1.073 \times 10^{5} \mathrm{~cm} . y r^{-1} \quad \text { for } I=46.38^{\circ} \text { and } 133.62^{\circ} \\
& =\alpha_{1} \frac{R_{\oplus}}{a} \times 4.509 \times 10^{4} \mathrm{~cm} \cdot \mathrm{yr}^{-1} \quad \text { for } I=73.15^{\circ} \text { and } 106.85^{\circ} \\
\frac{4 \delta \rho_{0}}{T_{0}} & =4 \frac{A}{B} \sin \delta \sin I \\
& =\alpha_{1} \frac{R_{\oplus}}{a} \times 1.395 \times 10^{4} \mathrm{~cm} \cdot \mathrm{yr}^{-1} \quad \text { for } I=63.43^{\circ} \text { and } 116.57^{\circ}
\end{aligned}
$$

These results underline that the inclinations around $46.38^{\circ}$ or $133.62^{\circ}$ have the capability to give the largest effects in a given observational time, as confirmed by the width of the resonances in Fig. 2. Equations (40) also show that low orbits (say $R_{\oplus}<a \lesssim 2 R_{\oplus}$ ) seem preferable in that they give larger $\delta \rho$ 's in a given observational time. [It is easy to see that the $a^{-1}$ dependence of $\delta \rho / T$ still applies for high, tidally perturbed orbits. This justifies our concentrating on low orbits, with negligible tidal effects.] However, equations (38),(39) show that the inclination $I$ must be fixed with a high precision, typically within less than one arc-minute if one wishes to make the fullest use of the observational time $T / 4$ which, for practical reasons, will probably not exceed $\sim 10$ years. The needed inclination can be computed thanks to the following asymptotic formulae:

$$
\begin{aligned}
& \left|I-I_{\text {pole }}\right| \approx\left(\frac{a}{R_{\oplus}}\right)^{7 / 2} 3.19^{\circ} \frac{1 \mathrm{yr}}{T_{ \pm}} \text {for } I_{\text {pole }}=46.38^{\circ} \text { or } 133.62^{\circ} \\
& \left|I-I_{\text {pole }}\right| \approx\left(\frac{a}{R_{\oplus}}\right)^{7 / 2} 2.41^{\circ} \frac{1 \mathrm{yr}}{T_{ \pm}} \text {for } I_{\text {pole }}=73.15^{\circ} \text { or } 106.85^{\circ} \\
& \left|I-I_{\text {pole }}\right| \approx\left(\frac{a}{R_{\oplus}}\right)^{7 / 2} 2.83^{\circ} \frac{1 \mathrm{yr}}{T_{0}} \quad \text { for } I_{\text {pole }}=63.43^{\circ} \text { or } 116.57^{\circ} .
\end{aligned}
$$


For instance a low satellite $\left(a \approx R_{\oplus}\right)$ will give rise to a perigee displacement $\delta \rho_{ \pm} \approx \alpha_{1} \times$ $10^{6} \mathrm{~cm}$ in $T_{ \pm} / 4=10 \mathrm{yr}$ if the inclination differs from the pole value $43.38^{\circ}$ (or $133.62^{\circ}$ ) by only $3.19^{\circ} / 40=4^{\prime} 47^{\prime \prime}$. An error of one arc-minute on $I$ would change the observational time and the perigee displacement by factors $\sim(0.8)^{ \pm 1}$.

The six resonant values of the inclination are solutions of the simple trigonometric equations $4-5 \sin ^{2} I+2 x \cos I=0$, where $x \in\{-1,0,1\}$, and do not depend on any experimental data. They will therefore enhance preferred-frame effects in the motion of the natural satellites of the different planets in the solar system (although tidal forces may not be negligible is some cases). However, none of the known natural satellites' orbits has an inclination close enough to one of the six poles. On the other hand, there are hundreds of artificial Earth satellites, and many of them would allow one to tighten the present bounds (9),(10) on $\alpha_{1}$ if the evolution of their perigee could be tracked at the centimeter level. For instance, the classes of satellites 'GPS BII' and 'GOES' would typically allow one to measure perigee radial displacements of $\alpha_{1} \times 3 \times 10^{5} \mathrm{~cm}$ in an observational time of $T / 4 \sim 15 \mathrm{yr}$. Even if these satellites could be tracked with sufficient precision, it would remain to see whether the effect of non-gravitational forces would allow one to make full use of such long data span. However, a statistical study of these $\sim 20$ satellites could probably allow a significant reduction of the sources of errors. The same remarks can be formulated about the classes of satellites 'GPS' and 'Glonass' whose perigees are deviated by $\sim \alpha_{1} \times 1.5 \times 10^{5} \mathrm{~cm}$ in $T / 4 \sim 8 \mathrm{yr}$. [There are also many low (i.e. fast) satellites, like 'Starlette' or 'Seasat', which give perigee oscillations of $\sim \alpha_{1} \times 3 \times 10^{4} \mathrm{~cm}$ in less than one year, but the air drag is very large for such low satellites.] The first Laser Geodynamical Satellite (LAGEOS I) is an interesting candidate, not only because its position has been laser tracked for years at the few centimeter level and because it is submitted to very small air drag, but also because several contributions to the eccentricity vector $\mathbf{e}$ are large in its case: $\mathbf{e}_{+}$gives a perigee deviation of $\alpha_{1} \times 4.2 \times 10^{4} \mathrm{~cm}$ in $T_{+} / 4 \sim 1.9$ years, $\mathbf{e}_{-}$a deviation of $\alpha_{1} \times 2.0 \times 10^{4} \mathrm{~cm}$ in $T_{-} / 4 \sim 5.3$ months, and $\mathbf{e}_{0}$ a deviation of $\alpha_{1} \times 8.8 \times 10^{3} \mathrm{~cm}$ in $T_{0} / 4 \sim 1.2$ years. The superposition of these three effects thus yields a complex signal which should, hopefully, be distinguishable from other, Newtonian, contributions. Unfortunately, the variations of the eccentricity $e$ of this satellite are not very precisely modelizable, as explained in Refs. [19,20]: it undergoes small oscillations of $\pm 3 \times 10^{-7}$, which correspond to perigee deviations of $\pm 4 \mathrm{~m}$. The recently launched second LAGEOS, which has an even more favorable inclination, might provide another interesting experimental probe of preferred-frame effects. Indeed, a duration of only $T_{+} / 4 \sim 1.3 \mathrm{yr}$ would suffice to observe a radial displacement of its perigee by $\delta \rho_{+} \sim \alpha_{1} \times 7 \times 10^{4} \mathrm{~cm}$. The best tool for tightening the present bounds (9), (10) on $\alpha_{1}$ would be a (naturally or artificially) drag-free satellite, launched on a favorably inclined orbit and tracked for years at the centimeter level (via laser ranging or onboard GPS receivers). Let us note that there are plans for launching in the near future drag-free satellites with GPS receivers onboard: Gravity Probe B (GPB) and the Satellite Test of the Equivalence Principle (STEP).

For completeness, we now briefly discuss how the above solution (35) for the eccentricity vector $\mathbf{e}$ is modified when tidal forces (which are very small for low satellites) are taken into account. The first (trivial) modification is of course that the disturbing function (15) now gives a contribution to the precession velocity $\langle\dot{\Omega}\rangle(12 \mathbb{1})$ of the orbital plane: 


$$
\langle\dot{\Omega}\rangle=-\frac{3}{4} n \cos I\left[2 J_{2}\left(R_{\oplus} / a\right)^{2}+N^{2} / n^{2}\right]+O\left(\alpha_{1} e\right)+O\left(e^{2}\right) .
$$

On the other hand, the effect of tidal forces in equations (12b) and (120) for $e$ and $\omega$ is somewhat more involved [even after averaging over monthly and yearly frequencies as explained in section II, Eq. (15)]. The vectorial equation satisfied by the eccentricity vector e in the orbital plane reads now

$$
\left\langle d^{\prime} \mathbf{e} / d t\right\rangle=\mathbf{c} \times(\mu \mathbf{e}+\mathbf{k} \times \mathbf{c})+\nu \mathbf{b} \times(\mathbf{a} \times \mathbf{e})+O\left(\alpha_{1} e^{2}\right)+O\left(e^{3}\right),
$$

where

$$
\mu \equiv \frac{3}{4} n\left[J_{2}\left(R_{\oplus} / a\right)^{2}\left(4-5 \sin ^{2} I\right)+2 N^{2} / n^{2}\right]
$$

is the analog of the angular frequency $\dot{\omega}_{N}$ of Eq. (30), and

$$
\nu \equiv \frac{15}{4 n} N^{2} \sin ^{2} I
$$

is an additional contribution due to tidal forces. The ratio $\nu / \mu$ can become positive and larger than unity when the inclination $I$ is very close to the well-known $J_{2}$-critical inclination $I_{0} \equiv \sin ^{-1}(2 / \sqrt{5}) \approx 63.43^{\circ}$ or $116.57^{\circ}\left(\right.$ e.g. $\left|I-I_{0}\right| \lesssim 4^{\prime}$ for $a \sim 2 R_{\oplus}$, and $\left|I-I_{0}\right| \lesssim 8^{\prime \prime}$ for $a \sim R_{\oplus}$ ). Equation (43) then formally exhibits an exponential blow up of the Newtonian eccentricity vector. This indicates that our simplified (linearized and time-averaged) treatment of the evolution of $\mathbf{e}$ becomes inadequate. In the following, we restrict our attention to the generic case $\nu / \mu \leq 1$. One finds that $\mathbf{e}_{N}$ has no longer a constant norm, as opposed to Eq. (23) and (35) above, but that it is now moving on an ellipse, whose axes are directed along $\mathbf{a}$ and $\mathbf{b}$ and have a ratio $e_{a} / e_{b}=(1-\nu / \mu)^{1 / 2}$ :

$$
\mathbf{e}_{N}=e_{a} \cos (\mu \sqrt{1-\nu / \mu} t+\mathrm{cst}) \mathbf{a}+e_{b} \sin (\mu \sqrt{1-\nu / \mu} t+\mathrm{cst}) \mathbf{b} .
$$

[Note that this ellipse reduces to a circle in the particular case of equatorial orbits $(I=0)$ that we considered in section II.] The solution of equation (43) can then be obtained in a geometrical manner similar to the one used in Eqs. (32)-(35). Let $\mathbf{e}_{i}$ denote a generalization of the Newtonian eccentricity vector $\mathbf{e}_{N}$ when the frequency $\mu$ is replaced by an arbitrary constant $\mu_{i}$ such that $\nu / \mu_{i} \leq 1$, i.e. $\mu_{i} \leq 0$ or $\mu_{i} \geq \nu$ (we shall see below that these values of $\mu_{i}$ are sufficient for our purpose). Like $\mathbf{e}_{N}$ in Eq. (46) above, $\mathbf{e}_{i}$ is moving on an ellipse in the orbital plane, with a non-uniform velocity which has the sign of $\mu_{i}$, and such that its components along $\mathbf{a}$ and $\mathbf{b}$ are oscillating with a constant frequency $\mu_{i}\left(1-\nu / \mu_{i}\right)^{1 / 2}$ and have an amplitude ratio $e_{a}^{i} / e_{b}^{i}=\left(1-\nu / \mu_{i}\right)^{1 / 2}$. [The particular cases of constant vectors along $\mathbf{a}$ or $\mathbf{b}$ are obtained respectively for $\mu_{i} \rightarrow 0^{-}$and $\left.\mu_{i}=\nu\right]$. The time derivative of a linear combination $\mathbf{e} \equiv \mathbf{e}_{N}+\sum_{i} \mathbf{e}_{i}$ then reads

$$
d^{\prime} \mathbf{e} / d t=\mathbf{c} \times\left(\mu \mathbf{e}+\sum_{i}\left(\mu_{i}-\mu\right) \mathbf{e}_{i}\right)+\nu \mathbf{b} \times(\mathbf{a} \times \mathbf{e}) .
$$

This has precisely the form of Eq. (43), with $\mathbf{k} \times \mathbf{c}$ having been decomposed as a sum of vectors $\left(\mu_{i}-\mu\right) \mathbf{e}_{i}$ moving on ellipses as described above. It is easy to check that any vector 
having a periodic motion in the orbital plane admits such a decomposition, and that the corresponding $\mu_{i}$ 's always satisfy the condition $\nu / \mu_{i} \leq 1$. In particular, one finds that the source term $\mathbf{k} \times \mathbf{c}$ can be decomposed as in Eq. (33), where $\mathbf{K}_{0}$ is still given by Eq. (34b), but where $\mathbf{K}_{ \pm}$have more complicated expressions

$$
\mathbf{K}_{ \pm} \equiv \cos \delta \frac{\dot{\Omega} / \mu_{\mp}+\cos I}{2-\nu / \mu_{\mp}}\left[-\sin (\Omega-\alpha) \mathbf{a}+\frac{\mu_{ \pm}}{\dot{\Omega}} \cos (\Omega-\alpha) \mathbf{b}\right]
$$

with

$$
\mu_{ \pm} \equiv \mp \dot{\Omega} \sqrt{1+(\nu / 2 \dot{\Omega})^{2}}+\nu / 2
$$

Note that these expressions for $\mathbf{K}_{ \pm}$reduce to those of Eq. (34a) when $\nu=0$, i.e. when tidal forces are neglected or for equatorial orbits. Hence the solution of Eq. (43) can be written simply as

$$
\mathbf{e}=\mathbf{e}_{N}+\alpha_{1} \frac{G m_{\oplus}}{4 a^{2} c^{2}} w\left(\frac{\mathbf{K}_{+}}{\mu-\mu_{+}}+\frac{\mathbf{K}_{0}}{\mu}+\frac{\mathbf{K}_{-}}{\mu-\mu_{-}}\right) .
$$

It is therefore of the same kind as Eq. (35), and there still exist poles for some particular values of the inclination, only slightly modified with respect to those of Fig. 2 for relatively low satellites. [For the same reason indicated above, our treatment becomes inadequate when $\mu \rightarrow 0^{+}$, formally corresponding to $(1-\nu / \mu)^{1 / 2}$ becoming large and pure imaginary.]

\section{PERTURBATIONS DUE TO THE ORBITAL VELOCITY OF THE EARTH AROUND THE SUN}

\section{A. Perturbations of the eccentricity vector}

In the previous sections, we have neglected the orbital velocity $\mathbf{v}_{\oplus}$ of the Earth around the Sun, but we shall see below that it can also lead to significant preferred-frame effects on artificial satellites, although they will typically be $12\left(\approx w / v_{\oplus}\right)$ times smaller than those proportional to $w$. The interest of effects involving $\mathbf{v}_{\oplus}$ is twofold. First of all, we shall see that arbitrarily small divisors can enhance the preferred-frame effects we are studying for any value of the inclination $I$ of the satellite's orbit (if the semi-major axis $a$ is chosen appropriately) instead of the discrete resonances displayed in Fig. 2. Moreover, the existence of a motion around the Sun (with well-defined amplitude and phase) is known for sure to be part of the 'absolute' velocities entering preferred-frame effects. By contrast, the identification of $\mathbf{w}$ with our velocity with respect to the cosmic microwave background is a specific assumption. Although this assumption is plausible on field-theoretical grounds, the bounds derived on $\alpha_{1}$ in the literature [3,6-9] and the discussions of the previous sections of the present paper are strongly dependent on it. It seems therefore of importance to determine limits on $\alpha_{1}$ by relying only on an unambiguously present velocity such as $\mathbf{v}_{\oplus}$.

It is straightforward to generalize the results of the previous section to the case of a velocity $\mathbf{v}_{\oplus}$ which is not constant, as opposed to $\mathbf{w}$. The equation satisfied by the eccentricity 
vector e is Eq. (30), but we concentrate now on the effects generated by the source term $\boldsymbol{\kappa}_{\oplus} \times \mathbf{c}$, where $\boldsymbol{\kappa}_{\oplus}$, given by Eq. (22), is a vector rotating with angular frequency $n_{\oplus} \equiv$ $\left(G m_{\oplus} / r_{\oplus \odot}^{3}\right)^{1 / 2}$. As in the previous section, it suffices to decompose $\boldsymbol{\kappa}_{\oplus} \times \mathbf{c}$ as a sum of constant-norm vectors rotating with constant angular frequencies to derive the contributions to e involving $v_{\oplus}$. Let us denote as $I_{\oplus}=23.5^{\circ}$ the inclination of the ecliptic with respect to the Earth's equatorial plane, and choose the origin of time at the vernal equinox. The source term $\boldsymbol{\kappa}_{\oplus} \times \mathbf{c}$ can then easily be decomposed as

$$
\boldsymbol{\kappa}_{\oplus} \times \mathbf{c}=-\alpha_{1} \frac{G m_{\oplus}}{4 a^{2} c^{2}} v_{\oplus}\left(\mathbf{K}_{++}+\mathbf{K}_{+-}+\mathbf{K}_{-+}+\mathbf{K}_{--}+\mathbf{K}_{0+}+\mathbf{K}_{0-}\right)
$$

where

$$
\mathbf{K}_{0 \pm} \equiv-\frac{1}{2} \sin I \sin I_{\oplus}\left[\cos \left(n_{\oplus} t\right) \mathbf{a} \mp \sin \left(n_{\oplus} t\right) \mathbf{b}\right]
$$

and if $s, s^{\prime}$ denote two independent signs $\left(s= \pm 1, s^{\prime}= \pm 1\right)$

$$
\mathbf{K}_{s s^{\prime}} \equiv s^{\prime} \frac{1+s \cos I}{2} \frac{1-s s^{\prime} \cos I_{\oplus}}{2}\left[\cos \left(s \Omega+s^{\prime} n_{\oplus} t\right) \mathbf{a}-\sin \left(s \Omega+s^{\prime} n_{\oplus} t\right) \mathbf{b}\right] \text {. }
$$

The vectors $\mathbf{K}_{0 \pm}$ are rotating with angular velocities $\mp n_{\oplus}$ in the orbital plane, whereas $\mathbf{K}_{s s^{\prime}}$ are rotating with angular velocities $-\left(s \dot{\Omega}+s^{\prime} n_{\oplus}\right)$ in this plane. These notations are chosen to simplify the expression of the eccentricity vector e written below; it generalizes the notations $\mathbf{K}_{ \pm}, \mathbf{K}_{0}$ introduced in section IV, which would be denoted $\mathbf{K}_{ \pm 0}, \mathbf{K}_{00}$ in the present convention. The eccentricity vector can then be immediately written as

$$
\begin{aligned}
\mathbf{e}=\mathbf{e}_{N}+\alpha_{1} \frac{G m_{\oplus}}{4 a^{2} c^{2}} v_{\oplus} & \left(\frac{\mathbf{K}_{++}}{\dot{\omega}_{N}+\dot{\Omega}+n_{\oplus}}+\frac{\mathbf{K}_{+-}}{\dot{\omega}_{N}+\dot{\Omega}-n_{\oplus}}\right. \\
& \left.+\frac{\mathbf{K}_{-+}}{\dot{\omega}_{N}-\dot{\Omega}+n_{\oplus}}+\frac{\mathbf{K}_{--}}{\dot{\omega}_{N}-\dot{\Omega}-n_{\oplus}}+\frac{\mathbf{K}_{0+}}{\dot{\omega}_{N}+n_{\oplus}}+\frac{\mathbf{K}_{0-}}{\dot{\omega}_{N}-n_{\oplus}}\right)
\end{aligned}
$$

to which should be added the contributions proportional to $w$ of Eq. (35). The solution (54) now exhibits resonances for any value of the inclination $I$ provided the semi-major axis $a$ is chosen appropriately. Figure 3 displays the values of $a$ and $I$ for which one of the divisors in Eq. (54) vanishes. Note that when $a$ tends formally towards 0, these curves tend towards the six poles found in section IV, because $\dot{\omega}_{N}$ and $\dot{\Omega}$ [Eqs. (31) and (29)] are proportional to $a^{-7 / 2}$ whereas $n_{\oplus}=2 \pi /(1 \mathrm{yr})$ is constant.

As in the previous section, the price to pay for a small divisor is the need for a correspondingly long time of observation, say $T / 4=\pi / 2\left(\dot{\omega}_{N} \pm \dot{\Omega} \pm n_{\oplus}\right)$, or $\pi / 2\left(\dot{\omega}_{N} \pm n_{\oplus}\right)$, depending upon the concerned divisor. The figure of merit $\delta \rho /(T / 4)$ of the different orbits can be computed like in Eqs. (40), and Figure 4 shows that the contributions $\mathbf{e}_{+-}$and $\mathbf{e}_{-+}$ give the best results, i.e. the largest perigee displacements in a given observational time. This is due to the rather small value of the inclination $I_{\oplus}=23.5^{\circ}$ of the ecliptic with respect to the equatorial plane, since $\mathbf{e}_{+-}$and $\mathbf{e}_{-+}$are precisely the only contributions which do not vanish as $I_{\oplus} \rightarrow 0$. The values of $a$ and $I$ for which these two main contributions diverge have been plotted in solid lines in Fig. 3 in order to distinguish them from those involving $\sin I_{\oplus}$ or $1-\cos I_{\oplus}$ (vanishing when $I_{\oplus} \rightarrow 0$ ) which have been plotted in dashed lines. 
The largest figure of merit displayed in Fig. 4 is obtained for $a \approx R_{\oplus}$ and $I=43.22^{\circ}$, and reads $4 \delta \rho_{+-} / T_{+-}=\alpha_{1} \times 8.62 \times 10^{3} \mathrm{~cm} . \mathrm{yr}^{-1}$. As expected, it is $\left(w / v_{\oplus} \approx\right) 12$ times smaller than the best result (40a) of section IV, which was obtained for $I=46.38^{\circ}$ (or $\left.133.62^{\circ}\right)$. However, the high-precision observation of a low orbit with an inclination close to $43.22^{\circ}$ could lead to the first experimental results about preferred-frame effects free from any assumption about the fundamental rest frame. [The satellite LACE happens to be precisely on such an orbit, but it is not drag-free.]

We have seen in section IV that all of the three contributions $\mathbf{e}_{+}, \mathbf{e}_{-}$and $\mathbf{e}_{0}$ (proportional to $w$ ) to the eccentricity vector e are a priori large for the orbit of LAGEOS I, thus giving rise to a complex signal in the evolution of the eccentricity vector. It is interesting to quote that the contribution $\mathbf{e}_{-+}$(proportional to $v_{\oplus}$ ) is also enhanced by a small divisor for this satellite, since the amplitude $\delta \rho_{-+}$is about $\alpha_{1} \times 2 \times 10^{3} \mathrm{~cm}$ in a typical observational time of $T_{-+} / 4 \sim 7$ months. It would be therefore interesting to analyze the LAGEOS I data to look for preferred-frame signals of both the $\mathbf{w}$ and the $\mathbf{v}_{\oplus}$ types [if it turns out feasible to extract such information in presence of the non-modelizable forces that act on the satellite].

A remarkable feature of Fig. 3 is the existence of maxima in the curves representing the loci of the $\mathbf{v}_{\oplus}$-type resonances in the $(I, a)$ plane. These maxima occur at $I=0$ or $180^{\circ}$ (for $a / R_{\oplus}=1.94,2.36$ and 2.65 ), $I=78.46^{\circ}$ or $101.54^{\circ}$ (for $a / R_{\oplus}=1.67$ ), and $I=90^{\circ}$ (for $a / R_{\oplus}=1.59$ ). The amplitude of the $\mathbf{v}_{\oplus}$-preferred-frame effects for orbits located close to these values of $I$ and $a$ would be therefore almost insensitive to injection errors or fluctuations in $I$. This suggests that these orbits might be especially robust tools for constraining this type of effects. The best choices would be the orbits located close to $\left(I=0, a=1.94 R_{\oplus}\right)$ or $\left(I=101.54^{\circ}, a=1.67 R_{\oplus}\right)$, which correspond to rather large figures of merit $4 \delta \rho / \alpha_{1} T$ in Fig. 4 (respectively $5.15 \times 10^{3} \mathrm{~cm} \cdot \mathrm{yr}^{-1}$ and $3.58 \times 10^{3} \mathrm{~cm} \cdot \mathrm{yr}^{-1}$ ).

\section{B. Along-track perturbations of the satellite}

We have not yet discussed the perturbation of the element $\sigma$, which is related to the angular position of the satellite at $t=0$. Using the expression of the disturbing function (13) in (12d), the secular evolution of $\sigma$ is given by

$$
\left\langle\frac{d \sigma}{d t}\right\rangle=\dot{\sigma}_{N}+\frac{\alpha_{1}}{4} \frac{n^{2} a}{c^{2}} \frac{\left[\mathbf{w}+\mathbf{v}_{\oplus}, \mathbf{c}, \mathbf{e}\right]}{e^{2}}-\frac{\alpha_{1} n}{c^{2}}\left(\mathbf{w}+\mathbf{v}_{\oplus}\right)^{2}+O\left(\alpha_{1} e\right),
$$

where $\dot{\sigma}_{N}$ is the Newtonian contribution due principally to tidal forces (15) and to the Earth's quadrupolar moment (14). The second term of the right-hand-side, though proportional to $1 / e$, does not lead to any interesting effect in the limit of a small eccentricity. Indeed, it precisely cancels a corresponding term in the secular evolution of $\omega$. In other words, the angular position of the satellite with respect to the ascending node is not affected by this type of contribution.

By contrast, the last term proportional to $\left(\mathbf{w}+\mathbf{v}_{\oplus}\right)^{2}=\mathbf{w}^{2}+\mathbf{v}_{\oplus}^{2}+2\left(\mathbf{w} \cdot \mathbf{v}_{\oplus}\right)$ induces interesting effects on the position of the satellite through the time variation of $\mathbf{w} \cdot \mathbf{v}_{\oplus}$. [The secular drift in $\sigma$ due to the constant term $\mathbf{w}^{2}+\mathbf{v}_{\oplus}^{2}$ is unobservable because, as we shall see below, it can be absorbed in a small renormalization of Newton's constant $G$.] Indeed, let us denote as before the right ascension and declination of $\mathbf{w}$ as $(\alpha, \delta)$, the orbital frequency 
of the Earth as $n_{\oplus}$, and the inclination of the ecliptic as $I_{\oplus}$. Equation (55) can then be rewritten as

$$
\langle\dot{\sigma}\rangle=\operatorname{cst}-2 \alpha_{1} n \frac{w v_{\oplus}}{c^{2}}\left[\frac{1+\cos I_{\oplus}}{2} \cos \delta \sin \left(n_{\oplus} t-\alpha\right)+O\left(1-\cos I_{\oplus}\right)+O\left(\sin I_{\oplus}\right)\right],
$$

where we have written down only the largest contribution. The integration of this equation shows that the longitude of the satellite is modulated by an oscillating term

$$
\delta \sigma=\alpha_{1} \frac{n}{n_{\oplus}} \frac{w}{c} \frac{v_{\oplus}}{c}\left(1+\cos I_{\oplus}\right) \cos \delta \cos \left(n_{\oplus} t-\alpha\right)
$$

corresponding to an along-track oscillation

$$
\delta x_{/ /}=a \delta \sigma \approx \alpha_{1}\left(R_{\oplus} / a\right)^{1 / 2} \cos \left(n_{\oplus} t-\alpha\right) \times 9.17 \times 10^{5} \mathrm{~cm} .
$$

The large amplitude, yearly periodicity, and weak altitude dependence $\left(\propto a^{-1 / 2}\right)$ of this effect makes it a promising way of improving the precision of measurement of $\alpha_{1}$ (maybe down to the $10^{-6}$ level). However, detailed numerical simulations are needed to assert whether the effect (58) can be separated from the other yearly perturbations.

Let us note finally that the results (57),(58) can be derived in a totally different way, by starting directly from the Lagrangians (1) and (11). The terms proportional to $\alpha_{1}$ can indeed be interpreted as a time-dependent renormalization of the gravitational constant [21,6] experienced by the satellite

$$
G(t) \equiv G\left[1-\frac{\alpha_{1}}{2 c^{2}}\left(\mathbf{w}+\mathbf{v}_{\oplus}\right)^{2}\right]
$$

Taking into account the adiabatic constancy of the Delaunay variable (or "action variable") $L \equiv\left[G(t) m_{\oplus} a(t)\right]^{1 / 2}$, Eq. (59) induces a variation of the semi-major axis

$$
a(t) \equiv a_{N}\left[1-\frac{\alpha_{1}}{2 c^{2}}\left(\mathbf{w}+\mathbf{v}_{\oplus}\right)^{2}\right]^{-1}
$$

In the limiting case of small eccentricity, the angular position $\theta$ of the satellite with respect to the perigee can be identified with the mean anomaly $\ell=\int n(t) d t+\sigma_{N}$, where $\sigma_{N}$ is a constant and where the orbital frequency $n(t)$ reads

$$
n(t)=\left(\frac{G(t) m_{\oplus}}{a(t)^{3}}\right)^{1 / 2}=n_{N}\left[1-\frac{\alpha_{1}}{2 c^{2}}\left(\mathbf{w}+\mathbf{v}_{\oplus}\right)^{2}\right]^{2} .
$$

Integrating Eq. (61) reproduces the result (57). Note that here again a very small instantaneous perturbation $O\left(v^{2} / c^{2}\right)$ has been enhanced by a large factor $n / n_{\oplus}$. As an aside, let us remark that another consequence of the time-dependent renormalization (59) of the gravitational constant is to cause a yearly "breathing" of the radius of the Earth, with associated yearly variations of its moment of inertia and of its angular velocity (see [3]). However, the amplitude of these variations, given the present limits on $\alpha_{1}$, are too small to be of observational significance, e.g. $\delta R_{\oplus}(t)=-\left(\partial \ln R_{\oplus} / \partial \ln G\right) \alpha_{1} R_{\oplus} \mathbf{w} \cdot \mathbf{v}_{\oplus}(t) / c^{2}<0.4 \mathrm{~mm}$. 


\section{CONCLUSIONS}

Artificial Earth satellites can be very useful tools to probe the field content of gravity, i.e. specifically whether it contains a vector or second tensor interaction leading to preferredframe effects in local gravitating systems. Thanks to the appearance of small divisors which enhance the preferred-frame effects on the eccentricity vector when the inclination and/or the semi-major axis of an orbit are chosen appropriately, it seems conceivable to tighten the present experimental bounds on the preferred-frame parameter $\alpha_{1}$ down to the $10^{-5}$ $10^{-6}$ level. What is needed are centimeter-level tracking data of a (naturally or artificially) drag-free satellite over time scales large enough to separate from Newtonian contributions the secular motion of the eccentricity vector induced by a non-zero $\alpha_{1}$. Among the class of zero-inclination (equatorial) orbits, geostationary ones are nearly optimal. Along-track oscillations of a satellite with yearly period constitute another promising way of measuring $\alpha_{1}$ around the $10^{-6}$ level. There is also a wide class of orbits for which preferred-frame effects on the eccentricity vector due to the orbital velocity of the Earth around the Sun are enhanced by small divisors. These could be used to obtain the first bounds on $\alpha_{1}$ independent of any hypothesis concerning the gravitationally preferred rest frame.

In this paper, we have presented an approximate analytical treatment of the dominant preferred-frame effects. The value of this treatment is mainly indicative, as a help for selecting the most favorable orbits. In practice, we advise to resort to direct numerical integration of the equations of motion (and, evidently, to a multiparameter fit to the experimental data). For the convenience of the interested reader, we end by giving the $\alpha_{1}$ (and for completeness $\alpha_{2}$ ) contributions to the equations of motion. Contrary to the rest of the paper, we have in mind here global (barycentric) equations of motion (written in a post-Newtonian coordinate system appropriate to the description of the entire solar system; see e.g. [22]). The relative acceleration of a satellite with respect to the Earth has the form

$$
\frac{d^{2} \mathbf{x}}{d t^{2}}=\mathbf{A}_{\mathrm{GR}}+\mathbf{A}_{\text {non-grav }}+\mathbf{A}_{\alpha_{1}}+\mathbf{A}_{\alpha_{2}}
$$

where the general relativistic geocentric acceleration (including a relativistic treatment of multipolar and tidal effects) will be found in full detail in Ref. [22], where $\mathbf{A}_{\text {non-grav }} \approx$ $\mathbf{F}_{\text {non-grav }} / m_{\text {sat }}$ denotes the acceleration induced by non-gravitational forces, and where (to lowest order in the deviation from general relativity, and for $m_{\mathrm{sat}} \ll m_{\oplus}, r \ll r_{\oplus \odot}$ )

$$
\begin{aligned}
\mathbf{A}_{\alpha_{1}}= & \alpha_{1} \frac{G m_{\oplus}}{2 r^{2} c^{2}}\left\{w_{\oplus}^{2} \mathbf{n}-\left(\mathbf{n} \times \mathbf{w}_{\oplus}\right) \times \mathbf{v}\right\} \\
& +\alpha_{1} \frac{G m_{\odot}}{2 r_{\oplus \odot}^{2} c^{2}}\left\{\left(\mathbf{n}_{\oplus \odot} \times \mathbf{w}\right) \times \mathbf{v}+\left(2\left[\frac{E_{\oplus}^{\mathrm{grav}}}{m_{\oplus}}\right]+\frac{G m_{\oplus}}{r}\right) \mathbf{n}_{\oplus \odot}\right\}, \\
\mathbf{A}_{\alpha_{2}}= & -\alpha_{2} \frac{G m_{\oplus}}{2 r^{2} c^{2}}\left\{\left(\mathbf{n} \times \mathbf{w}_{\oplus}\right)^{2} \mathbf{n}+2\left(\mathbf{n} \cdot \mathbf{w}_{\oplus}\right)\left(\mathbf{n} \times \mathbf{w}_{\oplus}\right) \times \mathbf{n}\right\} \\
& -\alpha_{2} \frac{G m_{\odot}}{2 r_{\oplus \odot}^{2} c^{2}}\left\{\frac{4}{3}\left[\frac{E_{\oplus}^{\mathrm{grav}}}{m_{\oplus}}\right] \mathbf{n}_{\oplus \odot}+\frac{G m_{\oplus}}{r}\left(\mathbf{n} \times \mathbf{n}_{\oplus \odot}\right) \times \mathbf{n}\right\} .
\end{aligned}
$$

In these formulae, $r$ and $\mathbf{v}$ denote the radial distance and the velocity of the satellite with respect to the Earth, $\mathbf{n}$ the unitary vector directed from the Earth to the satellite, $\mathbf{n}_{\oplus \odot}$ the 
unitary vector directed from the Earth to the Sun [23], and $\mathbf{w}_{\oplus}=\mathbf{w}+\mathbf{v}_{\oplus}$ the absolute velocity of the Earth with respect to the gravitationally preferred rest frame, $\mathbf{v}_{\oplus}$ being its orbital velocity around the Sun. For conceptual clarity we have indicated within square brackets the contributions of $\alpha_{1}$ and $\alpha_{2}$ due to the violation of the strong equivalence principle, i.e. the terms generated by expanding the $\eta$-dependent term coming from the Lagrangian $L_{\beta, \gamma, \eta}$, Eq. (2). The different terms of Eqs. (63) are classified by order of decreasing magnitude. [The even smaller contributions due to the coupling of the satellite to the Earth's intrinsic angular momentum are given in Eq. (9.20) of Ref. [3]; they cause an additional secular precession of the satellite's orbit which is independent of the absolute velocity with respect to the preferred frame.]

The contribution proportional to $w_{\oplus}^{2} \mathbf{n}$ in Eq. (63a) is responsible for the along-track perturbations of the satellite studied in section V.B above, whereas the one proportional to $\left(\mathbf{n} \times \mathbf{w}_{\oplus}\right) \times \mathbf{v}$, together with the contribution proportional to $\left(G m_{\oplus} / r\right) \mathbf{n}_{\oplus \odot}$ in the second line, is responsible for the perigee displacements studied in the rest of the paper. Note that the $\left(G m_{\oplus} / r\right) \mathbf{n}_{\oplus \odot}$ term has the same form as the one due to a violation of the strong equivalence principle [with an opposite sign: $2 E_{\oplus}^{\text {grav }} / m_{\oplus} c^{2} \approx-9.2 \times 10^{-10}, G m_{\oplus} / a c^{2} \approx\left(R_{\oplus} / a\right) \times 7.0 \times$ $\left.10^{-10}\right]$. Actually, neither of these two terms is of much observational significance, as their contributions are much smaller than that due to the $\left(\mathbf{n} \times \mathbf{w}_{\oplus}\right) \times \mathbf{v}$ term.

The force proportional to $\left(\mathbf{n}_{\oplus \odot} \times \mathbf{w}\right) \times \mathbf{v}$ in the second line of Eq. (63a) has not been considered in the rest of the paper. It arises in the connection between the locally inertial geocentric frame used in the body of the paper and the global barycentric one used here, and has the form of a Coriolis force. This Coriolis force acts also on gyroscopes (including the spinning Earth) and adds up to several other relativistic effects causing a universal precession $\boldsymbol{\Omega}$ of gyroscopes and satellite's orbits with respect to the barycentric frame (see section 9.1 of [3] and [22]). The $\alpha_{1}$-dependent contribution to $\Omega$ reads

$$
\mathbf{\Omega}_{\alpha_{1}}=\alpha_{1} \frac{G m_{\odot}}{4 r_{\oplus \odot}^{2} c^{2}} \mathbf{n}_{\oplus \odot} \times \mathbf{w}
$$

Although the time dependence of $\boldsymbol{\Omega}_{\alpha_{1}}$, via $\mathbf{n}_{\oplus \odot}(t)$, might in principle allow one to separate it from the other relativistic contributions to $\Omega$ (when discussing observables related to the global barycentric frame), its magnitude is too small to be of observational significance. [It yields a yearly oscillation of the satellite with respect to the barycentric frame which is smaller than $\left(a / R_{\oplus}\right) \times 0.1 \mathrm{~mm}$.] 


\section{REFERENCES}

[1] T. Damour and G. Esposito-Farèse, Class. Quantum Grav. 9, 2093 (1992).

[2] C.M. Will and K. Nordtvedt, Astrophys. J. 177, 757 (1972).

[3] C.M. Will, Theory and Experiment in Gravitational Physics (Cambridge University Press, Cambridge, 1981, revised 1993); Int. J. Mod. Phys. D 1, 13 (1992).

[4] K. Nordtvedt, Astrophys. J. 320, 871 (1987); beware that the latter paper uses a nonstandard normalization for $\alpha_{2}: \alpha_{2}^{\mathrm{N}}=\frac{1}{2} \alpha_{2}^{\text {standard }}$.

[5] In the present paper, we shall limit our consideration to the four post-Newtonian parameters $\beta, \gamma, \alpha_{1}, \alpha_{2}$, with special attention to $\alpha_{1}$. Although extended versions of the parametrized post-Newtonian (PPN) formalism have been defined, containing up to ten PPN parameters, the extra six parameters seem to be less well-rooted in plausible alternative field theories of gravitation.

[6] K. Nordtvedt and C.M. Will, Astrophys. J. 177, 775 (1972).

[7] R.J. Warburton and J.M. Goodkind, Astrophys. J. 208, 881 (1976).

[8] R.W. Hellings, in General Relativity and Gravitation, edited by B. Bertotti, F. de Felice and A. Pascolini, (D. Reidel Publishing Company, Dordrecht, 1984), pp. 365-385.

[9] T. Damour and G. Esposito-Farèse, Phys. Rev. D 46, 4128 (1992).

[10] T. Damour and K. Nordtvedt, Phys. Rev. Lett. 70, 2217 (1992); and Phys. Rev. D, 15 October 1993 (in press).

[11] G.F. Smoot et al., Astrophys. J. Lett. 371, L1 (1991).

[12] Actually, as we are interested in probing possible small deviations from general relativity $\left(\left|\alpha_{1}\right|<5 \times 10^{-4},|\gamma-1|<3 \times 10^{-3}, \ldots\right)$ it would (iteratively) suffice, even when dealing with orbital-period effects, to convert coordinate quantities into observable ones by interpreting them within a curved spacetime endowed with the general relativistic metric $g_{\mu \nu}^{\mathrm{GR}}$, without worrying about the $\alpha_{1}$-dependence (and $(\gamma-1)$-dependence, $\left.\ldots\right)$ of the coordinate/observable conversion.

[13] We follow the standard astronomical conventions in denoting by $\delta$ the declination (angle above the Earth equator; $\delta=+\pi / 2$ for the North pole) and by $\alpha$ the right ascension (longitude around the Earth polar axis counted from the vernal point).

[14] T. Damour and G. Schäfer, Phys. Rev. Lett. 66, 2549 (1991).

[15] D. Brouwer and G.M. Clemence, Methods of Celestial Mechanics (London: Academic Press, New York, 1961). Actually, there is a subtlety in the application of the traditional method of variation of the elements because the disturbing function depends also on velocities. One has the choice of working either with "osculating elements" (functions of positions and velocities) or with "contact" ones (functions of positions and canonical momenta); see V.A. Brumberg, Essential Relativistic Celestial Mechanics (Adam Hilger, Bristol, 1991). When considering first-order secular perturbations the two definitions give the same results, apart from a constant shift in the linear drift of $\sigma$ coming from a different definition of an (averaged) Newtonian orbital period.

[16] Within the present approach, the center of mass is defined using the standard Newtonian formulas in the preferred frame.

[17] To simplify our rough analytical estimates we assume that the $\sqrt{N}$ improvement due to the number of individual measurements just compensates the difficulty of determining the center of a circle from a limited segment ( $\sim$ a quarter $)$. 
[18] K. Nordtvedt, Phys. Rev. D 7, 2347 (1973); see Eqs. (28),(29).

[19] D.E. Smith and P.J. Dunn, Geophys. Research Lett. 7, 437 (1980).

[20] A. Milani, A.M. Nobili and P. Farinella, Non-gravitational Perturbations and Satellite Geodesy (Adam Hilger, Bristol, 1987).

[21] C.M. Will, Astrophys. J. 169, 141 (1971).

[22] T. Damour, M. Soffel and C. Xu, General-relativistic celestial mechanics IV. Theory of satellite motion, Phys. Rev. D, in press; see also T. Damour, M. Soffel and C. Xu, Phys. Rev. D 47, 3124 (1993) for a discussion of the universal Coriolis force (in general relativity) and the associated gravitational Larmor theorem.

[23] Note that this convention is opposite to the notation $\mathbf{n}_{A B}=\left(\mathbf{x}_{A}-\mathbf{x}_{B}\right) / r_{A B}$ used in Ref. [1] 


\section{FIGURES}

FIG. 1. Definition of the orbital elements e, $I, \Omega, \omega$, and of the rotating orthonormal basis $(\mathbf{a}, \mathbf{b}=\mathbf{c} \times \mathbf{a}, \mathbf{c})$ linked with the orbital plane. In the limit of a small eccentricity $e$, the angle between the perigee and the position of the satellite at $t=0$ can be identified with the orbital element $\sigma$.

FIG. 2. Expected radial displacements of the perigee as functions of the inclination of the orbital plane. The solid line correspond to the contribution $\delta \rho_{+}$, the dashed line to $\delta \rho_{-}$, and the dotted line to $\delta \rho_{0}$.

FIG. 3. Values of the semi-major axis of the satellite's orbit for which the displacement $\delta \rho$ of the perigee has a resonance, as functions of the inclination of the orbital plane. The bold lines correspond to the six poles shown on Fig. 2, which exist for any value of $a$ (small enough for tidal forces to be negligible). The solid lines correspond to effects proportional to $v_{\oplus}$ which do not vanish when the inclination $I_{\oplus}$ of the ecliptic with respect to the equatorial plane is neglected. The dashed lines correspond to the vanishing effects when $I_{\oplus} \rightarrow 0$. The dotted lines correspond to unphysical values of $a<R_{\oplus}$.

FIG. 4. Figure of merit of the resonant preferred-frame effects corresponding to the orbits of Fig. 3, i.e. ratio of the expected radial displacement $\delta \rho$ of the perigee by the typical observational

time $T / 4$. The plain lines correspond to the contribution $\delta \rho_{+ \pm}$, the dashed lines to $\delta \rho_{- \pm}$, and the dotted lines to $\delta \rho_{0 \pm}$. 
This figure "fig1-1.png" is available in "png" format from: http://arxiv.org/ps/gr-qc/9311034v1 
This figure "fig1-2.png" is available in "png" format from: http://arxiv.org/ps/gr-qc/9311034v1 


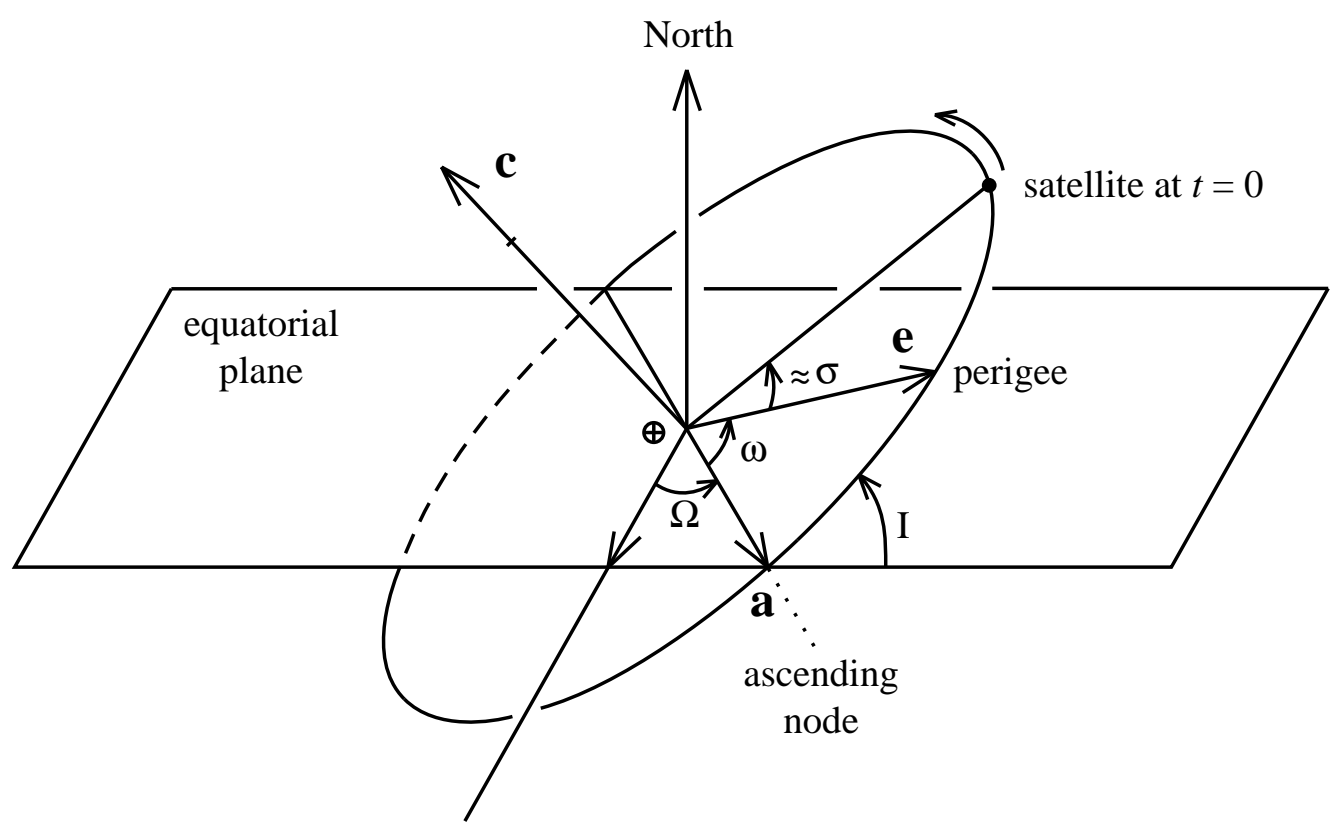

direction of reference

$$
(\alpha, \delta)=(0,0)
$$

Figure 1

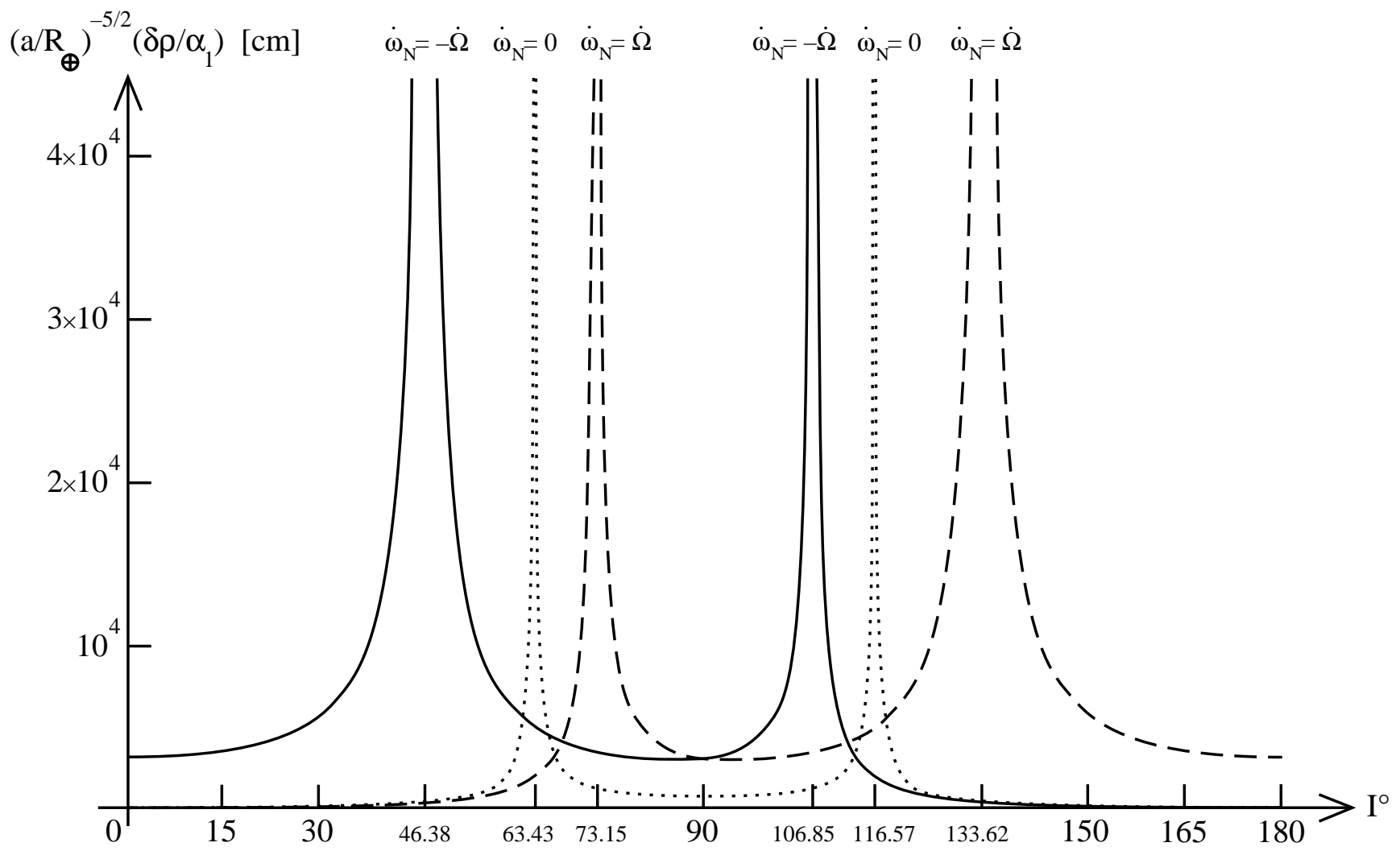

Figure 2 
